\title{
Postoperative Evaluation of Patient Satisfaction and Tingling Sensation after Replantation Surgery without Nerve Repair for Complete Digital Amputation
}

\author{
Hisataka Takeuchi, MD ${ }^{1}$ Ryosuke Ikeguchi, MD, $\mathrm{PhD}^{1}$ Mutsumi Watanabe, MD ${ }^{1}$ \\ Tadashi Yasuda, MD, $\mathrm{PhD}^{2}$ Shuichi Matsuda, MD, $\mathrm{PhD}^{1}$
}

${ }^{1}$ Department of Orthopaedic Surgery, Kyoto University Graduate School of Medicine, Kyoto, Japan

2 Department of Orthopaedic Surgery, Kobe City Medical Center

General Hospital, Kobe, Japan

\begin{abstract}
Address for correspondence Hisataka Takeuchi, MD, Department of Orthopaedic Surgery, Kyoto University Graduate School of Medicine, Shogoin Kawaramachi 54, Sakyo-ku - ward, Kyoto 606-8507, Japan (e-mail: orangepple1981@gmail.com).
\end{abstract}

J Reconstr Microsurg Open 2017;2:e19-e22.

\begin{abstract}
Keywords

- fingertip amputation

Background When performing replantation surgery for complete fingertip amputation, we do not perform digital nerve repair. We hypothesized that this method would not decrease patient satisfaction.

Methods Between July 2011 and August 2013, we performed replantation surgery for 21 complete digital amputations in 18 patients. Digital nerves were not repaired for fingertip amputations. For proximal to distal interphalangeal joint amputations (proximal amputation), however, we repaired as many digital nerves as possible. We followed 17 replanted fingers in 14 patients (fingertip, 9 fingers in 9 patients; proximal, 8 fingers in 5 patients) for $>1$ year, performing retrospective evaluation of subjective outcomes via telephone surveys. Patient satisfaction and fingertip tactile sensation scores (FTSS) were rated on scales of 0 to 10; unpleasant sensations (paresthesia or dysesthesia) were also surveyed.

Results Mean patient satisfaction was significantly greater in the fingertip-amputation group than in the proximal-amputation group ( 9.4 and 7.6 , respectively), although mean FTSS did not show significant difference ( 6.0 and 3.6, respectively). Patients with proximal amputations had dysesthesia in three fingers, paresthesia in one finger, and no numbness in four fingers, whereas patients with fingertip amputations had dysesthesia in three fingers, paresthesia in four fingers, and no numbness in two fingers. Patients with fingertip amputation had significantly more unpleasant sensation than those with proximal amputations.

- replantation surgery

- nerve repair

Conclusion Although fingertip replantation without digital nerve repair causes postoperative tingling, it results in good patient satisfaction.
\end{abstract}

In distal finger replantation, repair of a crushed or avulsed amputated nerve can be technically challenging. It is controversial whether it is necessary to repair the amputated nerve because some fingertip sensation is spontaneously acquired without any nerve repair. ${ }^{1}$ In addition, there is some discrepancy between acquired sensation and patient received

August 3, 2016

accepted after revision

December 5, 2016
DOI http://dx.doi.org/

10.1055/s-0037-1598249.

ISSN 2377-0813.
Copyright $\odot 2017$ by Thieme Medical

Publishers, Inc., 333 Seventh Avenue,

New York, NY 10001, USA.

Tel: +1(212) 584-4662.
License terms

(c) $9 \ominus \$$ 
satisfaction on an empirical basis. The aim of this study was to evaluate fingertip sensation in the replanted fingers and patient satisfaction.

\section{Methods}

Between July 2011 and August 2014, we performed replantation for complete distal finger amputation of 21 fingers in 18 patients (14 males, 4 females; mean age, 45.7 years [range, 1-81 years]). Ten amputations in 10 patients were fingertip. According to Ishikawa's classification system, six occurred in subzone III and four in subzone IV. Eleven amputations (eight cases) were proximal; seven fingers (six cases) were amputated at the middle phalanx and four fingers (two cases) at the proximal phalanx. Injury types were sharp $(n=2)$, crush $(n=8)$, and avulsion $(n=11)$. The amputated fingers were the thumb $(n=1)$, index finger $(n=6)$, long finger $(n=6)$, ring finger $(n=4)$, and little finger $(n=4)$. The amputation occurred on the right hand in 8 (38\%) fingers and on the left hand in 13 (62\%).

Replantation was successful in 19 fingers in 16 patients but failed in 2 fingers in 2 patients. The two successfully replanted fingers in two cases were lost to 1-year follow-up. Thus, this retrospective study included 17 surviving fingers in 14 patients.

Surgeries were performed under axillary-block anesthesia. During debridement of the distal and proximal stumps, arteries and veins were prepared for anastomosis. The bones were fixed with two Kirschner wires. When the level of amputation was proximal to the distal interphalangeal (DIP) joint, flexor and extensor tendons were repaired. We used vein grafts from the ipsilateral palmar side of the distal forearms to repair defects of the arteries, and anastomosed as many arteries and veins as possible. When the amputation level was proximal to the DIP joint, all digital nerves were repaired. For fingertip amputations (amputation level distal to the DIP joint), no nerve repair was performed. After confirmation of capillary refill, we sutured the skin loosely with 5-0 nylon. After surgery, the patients stayed in the hospital for 1 week. During the hospital stay, patients stayed in bed as much as possible and kept the injured hand above the heart to prevent swelling. All patients were given urokinase and alprostadil alfadex daily for 7 days postoperatively.

Patients were retrospectively assessed at least 12 months after surgery. Included in the study were 17 surviving fingers in 14 patients. The following three subjective examinations were administered via telephone survey: presence of fingertip tingling (paresthesia or dysesthesia), fingertip tactile sensation score (FTSS), and postoperative patient satisfaction. FTSS and patient satisfaction were rated on scales from 0 (no sensation/unsatisfied) to 10 (the same sensation as a noninjured finger/ completely satisfied).

\section{Results}

Nineteen of 21 fingers survived; 2 failed because of insufficient circulation. - Table 1 shows, for both groups,
Table 1 Details of vessel and nerve repair

\begin{tabular}{|c|c|c|}
\hline & $\begin{array}{l}\text { Fingertip } \\
\text { amputation } \\
(n=9)\end{array}$ & $\begin{array}{l}\text { Proximal } \\
\text { amputation } \\
(n=8)\end{array}$ \\
\hline Mean age (y) & 43.2 & 52.2 \\
\hline \multicolumn{3}{|c|}{ Arteries anastomosed per finger } \\
\hline $\begin{array}{l}\text { Subzone III per finger } \\
\text { (range) }\end{array}$ & $1.4(1-2)$ & \multirow[t]{2}{*}{2.0} \\
\hline $\begin{array}{l}\text { Subzone IV per finger } \\
\text { (range) }\end{array}$ & 2.0 (_2_) & \\
\hline \multicolumn{3}{|l|}{ Veins anastomosed per finger } \\
\hline $\begin{array}{l}\text { Subzone III per finger } \\
\text { (range) }\end{array}$ & $1.6(0-4)$ & \multirow[t]{2}{*}{2.1} \\
\hline $\begin{array}{l}\text { Subzone IV per finger } \\
\text { (range) }\end{array}$ & $1.7(1-4)$ & \\
\hline Nerves repaired per finger & $0^{\mathrm{a}}$ & 2.0 \\
\hline
\end{tabular}

${ }^{a}$ No nerve repair was performed for fingertip amputation.

the mean age of patients as well as number of veins and arteries anastomosed per finger and number of nerves repaired per finger. Digital nerves were not repaired for fingertip amputations. Both nerves were repaired in proximal amputations. The mean follow-up durations were 24.5 months (range, 13-37 months) for fingertip amputations and 26.8 months (range, 15-34 months) in proximal amputations.

- Table 2 shows outcomes of fingertip tingling, patient satisfaction, and FTSS. Patient satisfaction was significantly higher in the fingertip-amputation group than in the proximal-amputation group $(p=0.03)$. There was no significant difference in fingertip tingling and FTSS between groups.

\section{Discussion}

Replantation surgeries for finger amputations are technically challenging. Especially, it is always difficult for fingertip

Table 2 Outcomes of fingertip tingling, patient satisfaction, and fingertip satisfaction subjective score

\begin{tabular}{|l|l|l|l|}
\hline & $\begin{array}{l}\text { Fingertip } \\
\text { amputations } \\
(\boldsymbol{n}=9)\end{array}$ & $\begin{array}{l}\text { Proximal } \\
\text { amputations } \\
(\boldsymbol{n}=8)\end{array}$ & $p$ Value \\
\hline Fingertip tingling & $7(78 \%)$ & $4(50 \%)$ & \\
\hline Paresthesia & 4 & 2 & \\
\hline Dysesthesia & 3 & 2 & \\
\hline No tingling & $2(22 \%)$ & $4(50 \%)$ & \\
\hline $\begin{array}{l}\text { Patient } \\
\text { satisfaction }\end{array}$ & $9.4 \pm 0.9$ & $7.6 \pm 1.9$ & 0.03 \\
\hline FTSS & $6.0 \pm 2.6$ & $3.6 \pm 2.9$ & \\
\hline
\end{tabular}

Abbreviation: FTSS, fingertip tactile sensation score. 
replantation to find vessel and nerve stumps. This reason consists of not only the smaller diameter of the stumps but the various types of neurovascular anatomy. We tried replantation surgeries only for subzones III and IV. Recently, Nam reported the three patterns of the fingertip arteries. The type III, which has three or more dominant arteries distal from the distal transverse palmer arch, was the most common type and its frequency decreased from the index finger to little finger. ${ }^{2}$ These anatomical knowledge has the possibility to extend the indication of the fingertip replantation surgeries.

For the success of the replantation surgeries, recovery of sensation is cruciate, so nerve repair should be performed. However, in many avulsion or crushed-fingertip amputations, repair of the digital nerve is difficult, and there is no consensus as to the necessity of nerve repair in fingertip replantation. ${ }^{2}$ Sebastin and Chung performed a systematic review of 30 articles (2,273 fingers) on fingertip replantation surgery. ${ }^{3}$ Sixteen of the 30 articles (926 fingers) referenced nerve repair, which was performed in 13 articles ( 778 fingers) not attempted in the other 3 articles (148 fingers). A comparison demonstrated no significant difference in postoperative sensation between nerve repair and no nerve repair. This similarity is attributable to the pure sensory nature of the digital nerves and the relatively short distance between the level of repair and the fingertip, which make spontaneous recovery of fingertip sensation predictable. Spontaneous neurotization was thought to be easier in younger than in older people. Wong et al compared the results of fingertip amputation surgeries between nerve-repair and no-nerverepair groups and showed no significant between-group differences in pain, tingling, two-point discrimination distance test, and Semmes-Weinstein monofilament test. ${ }^{4}$ Hirase, ${ }^{5}$ Yamano, ${ }^{6}$ and Dubert et al $^{7}$ reported similar results.

In the present study, although nerve repair was not performed in fingertip amputation, fingertip touch sensation recovered spontaneously to some degree, as evidenced by a mean FTSS of $6.0 \pm 2.6$. These results were consistent with the reports described previously. The patient satisfaction score of $9.4 \pm 0.9$ in the fingertip-amputation group demonstrated a high degree of satisfaction. However, tingling was persistent in seven (78\%) of nine fingers at final follow-up; in contrast, tingling persisted in four (50\%) of eight fingers in the proximal-amputation group, in which all digital nerves were repaired. To prevent postoperative fingertip tingling, we recommend repair of the amputated digital nerves.

FTSS of the proximal-amputation group was lower than that of the fingertip-amputation group at 1-year follow-up. This can be explained by the distance between the injured side and terminal receptor being longer in the proximalamputation group than in the fingertip-amputation group, requiring more time for nerve regeneration. Although nerve grafting is an important technique when the digital nerve is crushed or avulsed, we attempted direct repair of the injured nerve without nerve grafting even under those conditions. This may also explain the poor FTSS results in the proximalamputation group. Recently, good results about collagen nerve conduits for digital nerve repair were reported. ${ }^{8}$ These conduits can become the alternation to nerve grafting. Hattori et al reported the results of replantation surgery in proximal phalangeal amputations (15 fingers in 13 cases) in which all nerves were repaired. Postoperatively, mean Semmes-Weinstein monofilament test score was 4.31 , and the authors concluded that good results could be achieved with nerve repair in proximal amputation. ${ }^{9}$ They also reported the result of proximal phalangeal replantation, and postoperative total active motion was 75 to 100 degrees (mean, 81 degrees), and the range of motion of the proximal interphalangeal (PIP) joint was 27 degrees of extension and 41 degrees of flexion. In the present study, patient satisfaction score was significantly lower in the proximal-amputation group than in the fingertip-amputation group. This could have been due not only to poor recovery of fingertip sensation but also to postoperative range of motion and the number of injured fingers.

The present study had some limitations. First, to evaluate the requirement for nerve repair in fingertip amputations, the optimal study design would be a randomized controlled trial to compare two groups randomly divided into with/ without nerve repair. However, the type of nerve injury depends on the mechanism of amputation injury and may be able to be identified only during surgery. Second, we used a telephone survey to evaluate postoperative condition; objective methods would have made the study more reliable. There were some discrepancies between acquired sensation and patient satisfaction on an empirical basis. Although previous reports included more objective data than the present study, few studies showed patients satisfaction. In addition, the most important component of the postoperative results of replantation surgery is patient satisfaction.

We repaired an injured nerve when it was cut proximal to the DIP joint (proximal-amputation group). When the cut level was distal to the DIP joint (fingertip-amputation group), no repair was attempted. The fingertip-amputation group, in which no nerve repair had been performed, had a higher FTSS and postoperative satisfaction score. However, tingling tended to persist in the fingers of the fingertipamputation group. Tingling of the fingertip can result when the digital nerve is not repaired during fingertip-amputation surgery. Therefore, if the injured nerve is cleanly cut and not damaged, it is preferable to perform nerve repair to prevent fingertip tingling. Although, in most cases, the nerve injuries associated with amputations are crush- or avulsion-type injuries and impossible to repair, the present study has demonstrated that, even in such cases, good outcomes of patient satisfaction and fingertip sensation can be achieved.

\section{Conclusion}

We examined postoperative outcomes of replantation surgery between a fingertip-amputation group, in which no nerve repair was performed, and a proximal-amputation group, in which all digital nerves were repaired. Although replantation surgery without digital nerve repair for fingertip amputation resulted in a high incidence of postoperative tingling, a high level of patient satisfaction was achieved. 
e22 Postoperative Evaluation of Patient Satisfaction after Replantation Surgery Takeuchi et al.

\section{References}

1 Nam YS, Jun YJ, Kim IB, Cho SH, Han HH. Anatomical study of the fingertip artery in Tamai zone I: clinical significance in fingertip replantation.J Reconstr Microsurg 2016. Doi:10.1055/s-0036-1588005

2 Ozcelik IB, Tuncer S, Purisa H, et al. Sensory outcome of fingertip replantations without nerve repair. Microsurgery 2008;28(07): 524-530

3 Sebastin SJ, Chung KC. A systematic review of the outcomes of replantation of distal digital amputation. Plast Reconstr Surg 2011; 128(03):723-737

4 Wong C, Ho PC, Tse WL, Cheng S, Chan DK, Hung LK. Do we need to repair the nerves when replanting distal finger amputations? J Reconstr Microsurg 2010;26(05):347-354
5 Hirase Y. Salvage of fingertip amputated at nail level: new surgical principles and treatments. Ann Plast Surg 1997;38(02):151-157

6 Yamano Y. Replantation of the amputated distal part of the fingers. J Hand Surg Am 1985;10(02):211-218

7 Dubert T, Houimli S, Valenti P, Dinh A. Very distal finger amputations: replantation or "reposition-flap" repair? J Hand Surg [Br] 1997;22(03):353-358

8 Lohmeyer JA, Kern Y, Schmauss D, et al. Prospective clinical study on digital nerve repair with collagen nerve conduits and review of literature. J Reconstr Microsurg 2014;30(04):227-234

9 Hattori Y, Doi K, Hoshino S, et al. Long term results of digital replantation following complete amputation at the level of proximal phalanx. J Japanese Soc Reconstr Microsurg 2009;22:353-358 